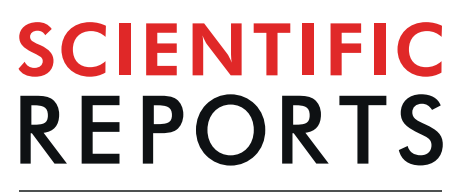

natureresearch

\title{
Factors associated with false negative interferon $-\gamma$ release assay results in patients with tuberculosis: A systematic review with meta-analysis
}

\author{
MariYamasue $\mathbb{C}^{1,3}$, Kosaku Komiya ${ }^{1,3^{*}}$, Yuko Usagawa ${ }^{1}$, Kenji Umeki ${ }^{1}$, Shin-ichi Nureki ${ }^{1}$, \\ Masaru Ando ${ }^{1}$, Kazufumi Hiramatsu ${ }^{1}$, Hideaki Nagai ${ }^{2}$ \& Jun-ichi Kadota ${ }^{1}$
}

Which factors are related to false negative results of the interferon- $\gamma$ release assay (IGRA) is unclear. This systematic review described the risk factors associated with false negative IGRA results. Two authors independently identified studies designed to evaluate risk factors for false negative IGRA results from PubMed, the Cochrane Register of Control Trial database, and EMBASE, accessed on October 22, 2018. Meta-analyses were conducted with random-effect models, and heterogeneity was calculated with the $l^{2}$ method. Of 1,377 titles and abstracts screened, 47 full texts were selected for review, and we finally included 17 studies in this systematic review. The most commonly studied risk factor (14 studies) was advanced age, followed by low peripheral lymphocyte counts ( 7 studies), and these factors were associated with false negative results even with different tuberculosis incidences (pooled odds ratio $2.06 ; 95 \% \mathrm{Cl}, 1.68-2.52$ in advanced age and $2.68 ; 95 \% \mathrm{Cl}, 2.00-3.61$ in low peripheral lymphocyte counts). Advanced age and low peripheral lymphocyte counts may be common risk factors for false negative IGRA results, suggesting that people with these factors need to be carefully followed, even if they have negative IGRA results.

Tuberculosis (TB) is one of the most serious infectious diseases contributing to worldwide morbidity and mortality ${ }^{1}$. The early diagnosis and treatment are effective strategies for preventing the spread of Mycobacterium tuberculosis infection. The M. tuberculosis-specific interferon- $\gamma$ release assay (IGRA) is widely considered to identify latent TB infection (LTBI) or to support the diagnosis of active TB infection as an adjunctive test ${ }^{2-4}$. Several risk factors for LTBI and the development of active TB are mentioned in the World Health Organization (WHO) guidelines. HIV infection, contacts bacteriologically confirmed pulmonary TB, initiating anti-TNF treatment, dialysis, organ or hematological transplant and silicosis carry a particularly high risk of TB infection ${ }^{5}$. Screening for LTBI in people with these factors is therefore strongly recommended to prevent the spread of TB infection.

The QuantiFERON Gold in-tube test (QFT-GIT) (Qiagen, Dusseldorf, Germany) as an enzyme-linked immunosorbent assay and T-SPOT.TB test (Oxford Immunotec, Oxford, UK) using an enzyme-linked immune spot (ELISPOT) method are mainly used as commercially available IGRAs. As there is no gold standard for the diagnosis of LTBI, the diagnostic accuracy has been studied using active TB cases. Nevertheless, the accuracy has not reached an adequate level yet. In fact, the pooled sensitivity of these assays for the diagnosis of culture-confirmed active TB has been reported to be $81 \%$ and $92 \%$ in QFT-GIT and T-SPOT, respectively, and approximately 8-19\% patients have negative IGRA results ${ }^{4}$.

False negative results prompt physicians to inappropriately end follow-up and abandon the consideration of prophylactic treatments in patients with possible LTBI. Therefore, analyzing the risk factors for false negative

\footnotetext{
${ }^{1}$ Department of Respiratory Medicine and Infectious Diseases, Oita University Faculty of Medicine, 1-1 Idaigaoka, Hasama-machi, Yufu, Oita, 879-5593, Japan. ${ }^{2}$ Center for Pulmonary Diseases, National Hospital Organization Tokyo National Hospital, 3-1-1 Takeoka, Kiyose, Tokyo, 204-8585, Japan. ${ }^{3}$ These authors contributed equally: MariYamasue and Kosaku Komiya. *email: komiyakh1@oita-u.ac.jp
} 
IGRA results is vital to identify patients who need careful follow-up despite negative results. Several risk factors for false negative IGRA results have been reported ${ }^{6-8}$, but there has been no review of these factors.

The present systematic review therefore assessed the risk factors associated with false negative IGRA results using published studies.

\section{Methods}

Search criteria. This systematic review was conducted according to the guidelines of the preferred reporting items for systematic reviews and meta-analyses (PRISMA) statement and Meta-analysis of observational Studies in epidemiology (MOOSE) guidelines ${ }^{9,10}$. Studies that evaluated the risk factors influencing false negative IGRAs results in patients with bacteriologically confirmed active TB were included. Due to the fact that risk factors for false negative IGRA results are confounded by other variables, we restricted inclusion to studies that performed statistical adjustments by a multivariate analysis in order to exclude low-quality studies.

We searched for studies using PubMed, Cochrane Central Register of Controlled Trials (CENTRAL) and the EMBASE database from August 1992 to October 2018. Combinations of the following search terms were applied: "positivity OR false negative” AND “interferon-gamma release assay OR ELISPOT OR QuantiFERON” (assessed on October 22, 2018). Publications written in languages other than English, studies published only in abstract form and studies in which active TB was not diagnosed by culture positivity, the results of two IGRAs were not described separately or statistical methods were not clearly stated were excluded. We also excluded the studies performed only in children.

The title, abstracts and full texts articles were screened and further evaluated by two authors (YM and KK) independently. Disagreements were resolved by the decision of a third reviewer (JK).

Data extraction. We extracted the following information from the included studies: study design, sample size, country in which the study was conducted, enrolled age groups, types of IGRAs (e.g. QuantiFERON or/ and ELISPOT), single or repeated IGRA testing, where the assay was performed (in-house or commercially) and history of TB and assessed the risk factors for false negative IGRAs results. Regardless of the significance of the results, potential risk factors for false negative IGRA results assessed in two or more studies were analyzed in this review.

We classified the countries where the studies were conducted according to the incidence of TB. The classification proposed by the WHO was used: low-incidence country, incidence of TB $<10$ new patients per 100,000 population each year; middle-incidence country, incidence of TB 10-100 new patients per 100,000 population each year; and high-incidence country, incidence of TB >100 new patients per 100,000 population each year ${ }^{1}$.

Assessing the risk of bias. The risk of bias was assessed according to the recommendations outlined in the Cochrane handbook for systematic reviews of interventions version 5.1.0 and MOOSE guidelines for the following items: selection, performance, detection, attrition and publication bias. Each study included in this systematic review was assessed for the quality based on biases using the modified Hayden's criteria ${ }^{11}$. We assessed the studies for the six factors related to potential biases, as follows: (1) study sample (e.g. source population clearly defined, study population described, and study population represents source population or population of interest), (2) participation rate, (3) analytical procedure clearly described, (4) outcome measurement (e.g. outcome defined and measured appropriately), (5) confounding measurement and accounting (e.g. confounders defined and measured as well as accounted for) and (6) analysis (e.g. analyses described, appropriate and provides sufficient presentation of data). Disagreements between the investigators were resolved by a review of the assessments to reach consensus.

Data analyses. Meta-analyses were conducted for outcomes with more than two raw data pools available from the included studies. Outcomes were pooled using Mantel-Haenszel risk ratios, and the precision of the estimates was expressed as the $95 \%$ confidence interval (CI). Statistical heterogeneity was assessed using the Higgins $I^{2}$ tests. A random-effects model was used when significant heterogeneity was found. Publication bias was assessed by an examination of funnel plots ${ }^{12}$. Statistical significance was defined by a p-value $<0.05$ for all analyses. The meta-analysis was performed with the Review Manager software program, ver. 5.3 (The Nordic Cochrane Centre, The Cochrane Collaboration, London).

\section{Results}

Database search and characteristics of the included studies. We identified 454, 280 and 643 studies through PubMed, the CENTRAL and the EMBASE, respectively. We excluded 1,330 studies as the abstract did not meet the inclusion criteria. We excluded 30 of the remaining 47 records after retrieving and inspecting the full text (Fig. 1). We finally included 17 studies in this systematic review: $14^{6,7,13-24}$ and 3 studies ${ }^{8,25,26}$ were retrospective and prospective observational studies, respectively. These published studies, 4 from low-incidence countries: United States of America $(n=2)^{6,22}$, Denmark $(n=1)^{13}$ and the EU $(n=1)^{14} ; 11$ from middle-incidence countries: South Korea $(\mathrm{n}=6)^{14,16-18,20,26}$ and China $(\mathrm{n}=4)^{8,13,15,21}$ and Japan $(\mathrm{n}=1)^{7}$; and 3 from high-incidence countries: Viet Nam $(n=1)^{25}$, Tanzania $(n=1)^{13}$ and Zambia $(n=1)^{23}$. The results from Denmark and Tanzania were reported collectively in one study ${ }^{13}$. Six studies evaluated risk factors for false negative results of IGRAs in a non-HIV population ${ }^{7,8,16,18,20,24}$ (Table 1). How many times the IGRA was performed per sample was not mentioned in any study included in this review.

Among the 17 studies included in our review, the gender, advanced age, low peripheral lymphocyte counts (including $\mathrm{CD}^{+}$T lymphocytes), HIV positivity, extrapulmonary TB and body mass index (BMI) were studied using a multivariate analysis in 3,14, 7, 4, 5 and 3 studies, respectively (Fig. 1). None of the 3 reports that studied gender, 5 of the 14 reports that studied advanced age, 4 of the 7 reports that studied low peripheral lymphocyte counts, 3 of the 4 reports that studied HIV positivity, all of the 5 reports ${ }^{15,19,21,24,26}$ that studied extrapulmonary 


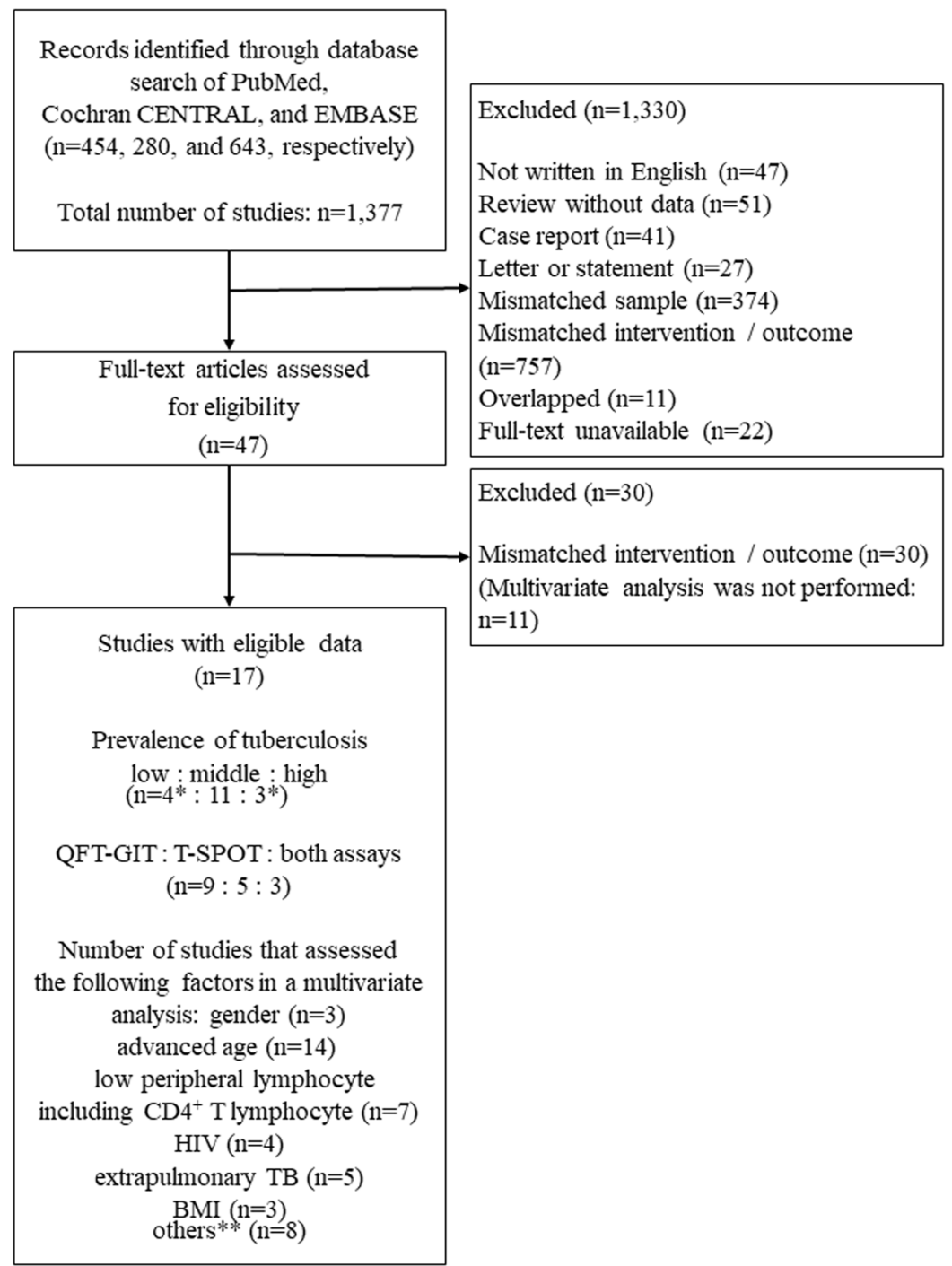

Figure 1. Flow diagram of the study selection. *Eighteen studies are not shown because the results from Denmark (low-incidence country) and Tanzania (high-incidence country) were reported collectively in a single study, so the total number of studies was 17.**Smoking, hospitalization, immunosuppressive conditions, immunosuppressive therapy, malignancy, CRP, low albumin, and HLA type (DRB1*0701 alleles): $\mathrm{n}=1$.

TB and 2 of the 3 reports that studied BMI as risk factors for false negative IGRA results provided the number of patients with false negative results in both the risk group and non-risk group. Other potential risk factors were reported but only in single studies. Therefore, we conducted a meta-analysis of the advanced age, low peripheral lymphocytes counts, HIV positivity, extrapulmonary TB and BMI.

Assessing the risk of bias. We evaluated the quality in all included studies using the modified Heyden's criteria (Table 2). The average number that met the six indicators for evaluating the potential bias among the studies was approximately 3.5. While the quality of study participation, analytical procedure, outcome measurement and analysis were mostly good to evaluate among studies, that of the participation rate and the confounding measurement and account were relatively poor. Only $7^{14,17,18,20,21,23,25}$ of 17 studies had a sufficient participation rate, and the rates in the others were under $50 \%$. For confounding measurement and accounting, eight studies did not assess adequately. The main reason for the low quality in this indicator was that the results of univariate analyses were not described, and possible confounding factors to be evaluated were not considered.

High heterogeneity was observed in the studies that evaluated lymphocyte counts, HIV positivity, extrapulmonary TB and BMI as risk factors (Figs. 2-6). As for publication bias, there appeared to be funnel plot asymmetry for low lymphocyte counts, HIV positivity, extrapulmonary TB and BMI but not advanced age, suggesting a low possibility of publication bias (see Supplementary Fig. S1). Due to the small number of studies included in each meta-analysis, Sterne's test was not appropriate for detecting funnel plot asymmetry ${ }^{12}$. 


\begin{tabular}{|c|c|c|c|c|c|c|c|c|c|c|c|}
\hline Author, year & Nationality & Study design & $\begin{array}{l}\text { Sample } \\
\text { size }\end{array}$ & $\begin{array}{l}\text { Age, years } \\
\text { median (range, } \\
\text { IQR or } \pm \text { SD) }\end{array}$ & Male (\%) & HIV (\%) & ЕРТВ (\%) & $\begin{array}{l}\text { History of } \\
\text { TB (\%) }\end{array}$ & IGRA & $\begin{array}{l}\text { True-positive } \\
(\%)\end{array}$ & $\begin{array}{l}\text { False-negative } \\
(\%)\end{array}$ \\
\hline Kim 2018 & South Korea & retrospective & 163 & $55(65<35 \%)$ & $85(52.1)$ & $1(0.6)$ & $163(100)$ & $18(11.0)$ & QFT-GIT & 69.9 & 28.8 \\
\hline Yang 2018 & China & retrospective & 2,425 & $43.6 \pm 18.5$ & $1,561(64)$ & 0 & $143(5.9)$ & nd & T-SPOT & 75.1 & 24.9 \\
\hline Nugyen 2018 & USA & retrospective & 1,487 & 47 (IQR: 30-61) & $942(63.3)$ & $90(13.2)$ & $196(13.2)$ & $32(2.2)$ & \begin{tabular}{|l}
$875(65.4)$ in \\
QTF-GIT \\
463(34.6) in \\
T-SPOT \\
\end{tabular} & \begin{tabular}{|l}
87.7 in total \\
nd in QFT- \\
GIT \\
nd in T-SPOT \\
\end{tabular} & $\begin{array}{l}12.3 \text { in total } \\
12.2 \text { in QFT-GIT } \\
16.4 \text { in T-SOPT }\end{array}$ \\
\hline Di 2018 & China & retrospective & 98 & $\begin{array}{l}\text { nd } \\
(<3021.4 \%, \\
30-6050.0 \%, \\
60<28.6 \%) \\
\end{array}$ & $55(56.1)$ & nd & $69(70.4)$ & 0 & T-SPOT & 83.7 & 16.3 \\
\hline Lian 2017 & China & retrospective & 556 & \begin{tabular}{|l|}
44.2 \\
(range 0.75-85) \\
\end{tabular} & $333(59.9)$ & $2(0.4)$ & $358(64.4)$ & nd & T-SPOT & 86.2 & 13.8 \\
\hline Kown 2015 & South Korea & retrospective & 1,264 & \begin{tabular}{|l}
50.3 (IQR: \\
$35-69)$ \\
\end{tabular} & $718(56.8)$ & 0 & $158(12.5)$ & $165(13.1)$ & QFT-GIT & 85.6 & 14.4 \\
\hline Choi 2015 & USA & retrospective & 300 & $48.1 \pm 22.1$ & $195(65.0)$ & $18(6)$ & $52(17.3)$ & nd & \begin{tabular}{|l|} 
QFT-GIT \\
QFT-2G \\
\end{tabular} & 70.3 & 29.7 \\
\hline Visser 2015 & Europe & retrospective & 664 & $\begin{array}{l}41 \text { (IQR: } 30-53) \\
\text { in QFT } \\
41 \text { (IQR: 28-56) } \\
\text { in T-SPOT }\end{array}$ & nd & nd & nd & nd & $\begin{array}{l}\text { QTF-GIT } \\
\text { T-SPOT }\end{array}$ & $\begin{array}{l}66.6 \text { in total } \\
\text { nd in QFT-GIF } \\
\text { nd in T-SOPT } \\
\text { nd in both } \\
\text { IGRA }\end{array}$ & $\begin{array}{l}33.2 \text { in total } \\
22.7 \text { in QFT-GIT } \\
4.8 \text { in T-SPOT } \\
1.0 \text { in both IGRA }\end{array}$ \\
\hline Pan 2014 & China & prospective & 774 & 45 (range 11-91) & $465(60.1)$ & 0 & $244(31.5)$ & nd & T-SPOT & \begin{tabular}{|l|}
89.9 in total \\
91.3 in PTB \\
86.9 in EPTB \\
\end{tabular} & \begin{tabular}{|l|}
10.1 in total \\
8.7 in PTB \\
13.1 in ЕPTB \\
\end{tabular} \\
\hline Lee 2013 & South Korea & prospective & 128 & $65<21.1 \%$ & $53(41.4)$ & $5(3.9)$ & $84(66)$ & $13(10.2)$ & T-SPOT & 82.8 & 17 \\
\hline Joen 2013 & South Korea & retrospective & 168 & $54.8 \pm 20.1$ & $102(60.7)$ & 0 & $10(5.9)$ & $3(1.8)$ & QFT-GIT & 76.8 & 23.2 \\
\hline Kim 2013 & South Korea & retrospective & 44 & $64 \pm 19.0$ & $17(39)$ & $2(4.5)$ & nd & nd & QFT-GIT & 68.2 & 16 \\
\hline Aabye 2012 & $\begin{array}{l}\text { Denmark } \\
\text { Tanzania }\end{array}$ & retrospective & $\begin{array}{l}34 \\
172\end{array}$ & $\begin{array}{l}50 \text { (range 23-76) } \\
32 \text { (range 15-84) }\end{array}$ & $\begin{array}{l}24(70.5) \\
64(37.2)\end{array}$ & \begin{tabular}{|l|}
4 \\
$(8)$ \\
$75(43.6)$ \\
\end{tabular} & \begin{tabular}{|l|}
8 \\
$(23.1)$ \\
nd
\end{tabular} & 0 & QFT-GIT & $\begin{array}{l}64.7 \\
71.5\end{array}$ & $\begin{array}{l}11.3 \\
3.8\end{array}$ \\
\hline Hang 2011 & Viet Nam & prospective & 504 & \begin{tabular}{|l|}
38.8 \\
(IQR: 29.2-50.8)
\end{tabular} & $399(79.2)$ & $44(8.7)$ & 0 & nd & QFT-GIT & 92.3 & 4.8 \\
\hline Kim 2011 & South Korea & retrospective & 362 & 49 (IQR: 16-94) & 197 (54.4) & 0 & 0 & $55(15.2)$ & QFT-GIT & 85.9 & 14.1 \\
\hline Komiya 2010 & Japan & retrospective & 215 & 67 (IQR: 50-79) & $156(73)$ & 0 & 0 & nd & $\begin{array}{l}\text { QFT-G } \\
\text { ELISPOT }\end{array}$ & $\begin{array}{l}74 \\
93\end{array}$ & $\begin{array}{l}23 \\
7.4 \\
\end{array}$ \\
\hline Raby 2008 & Zambia & retrospective & 112 & 31 (IQR: 25-36) & $71(63)$ & $59(52.7)$ & nd & $20(18)$ & QFT-GIT & 74 & 12 \\
\hline
\end{tabular}

Table 1. Characteristics of the studies included in this systematic review. EPTB, extrapulmonary TB; nd, not described.

Gender. Male gender was studied as a risk factor for false negative results using a multivariate analysis in three studies $^{7,13,23}$. No study showed a significant relationship between male gender and false negative IGRA results (see Supplementary Table S1).

Advanced age. Pooled odds ratio (pooled OR: 2.06, 95\% CI 1.68-2.52) of advanced age calculated with raw data available in 5 of the 14 included studies ${ }^{6,8,15,20,22}$ showed a significant relationship between advanced age and false negative results with very low heterogeneity (Fig. 2). Eight of the 14 studies ${ }^{8,14,16,20-22,24,25}$ found that advanced age was significantly associated with false negative results in a multivariate analysis (see Supplementary Table S2). However, definitions of advanced age varied among the enrolled studies. While Kwon et al. compared groups $<65$ and $\geq 65$ years old ${ }^{20}$, Nugyen et al. set 60 years old as the cut-off value ${ }^{22}$. Pan et al. used the median age as the cut-off value $^{8}$, and four studies did not clearly state the cut-off value ${ }^{14,21,24,25}$. Joen et al. compared the risk of false negative results by age group and showed that the rate increased in the population over 40 years of age ${ }^{16}$.

Low peripheral lymphocyte counts. Peripheral lymphocyte counts and CD $4^{+} \mathrm{T}$ lymphocyte counts were evaluated as a risk factor using a multivariate analysis in five $e^{7,16-18,20}$ and two studies ${ }^{23,24}$, respectively; most of the studies were from middle-incidence countries. Low peripheral lymphocyte counts were significantly associated with false negative results in pooled analyses (pooled odds ratio: 2.68, 95\% CI 2.00-3.61) using available data from 4 of the 7 studies ${ }^{7,17,20,23}$ with moderate heterogeneity (Fig. 3). Five studies ${ }^{7,16,17,20,23}$ reported that low peripheral lymphocyte counts were significantly associated with false negative results in individual multivariate analyses (see Supplementary Table S3). Three of the five studies performed the analysis in a non-HIV population ${ }^{7,16,20}$, and cut-off values varied among studies $7,16,17,20,23$. Raby et al. assessed this factor in the HIV population and reported that a low $\mathrm{CD} 4^{+}$lymphocyte count $(<200 / \mu \mathrm{l})$ was a risk factor ${ }^{23}$, whereas Yang et al. reported that $\mathrm{CD}^{+}$lymphocyte counts were not a significant risk factor ${ }^{24}$.

HIV positivity. Four studies evaluated the association between HIV positivity and false-negative results of IGRA $^{6,13,22,25}$. Two studies were published from countries with a high incidence of both HIV infection and $\mathrm{TB}^{13,25}$. 


\begin{tabular}{|c|c|c|c|c|c|c|}
\hline Author, year & $\begin{array}{l}\text { Study } \\
\text { sample }\end{array}$ & $\begin{array}{l}\text { Participation } \\
\text { rate }\end{array}$ & $\begin{array}{l}\text { Analytical procedure } \\
\text { clearly described }\end{array}$ & $\begin{array}{l}\text { Outcome } \\
\text { measurement }\end{array}$ & $\begin{array}{l}\text { Confounding measurement } \\
\text { and accounting for } \\
\text { confounders }\end{array}$ & Analysis \\
\hline Kim 2018 & - & - & + & + & + & + \\
\hline Yang 2018 & + & - & + & + & - & + \\
\hline Nugyen 2018 & + & - & nd & nd & - & - \\
\hline Di 2018 & + & - & + & + & - & + \\
\hline Lian 2017 & + & + & + & + & + & + \\
\hline Kown 2015 & + & + & + & + & + & - \\
\hline Choi 2015 & + & - & + & + & - & - \\
\hline Visser 2015 & + & + & nd & nd & - & + \\
\hline Pan 2014 & + & - & + & + & + & + \\
\hline Lee 2013 & - & - & + & + & + & + \\
\hline Joen 2013 & + & - & + & + & nd & - \\
\hline Kim 2013 & - & + & - & - & + & + \\
\hline Aabye 2012 & - & - & + & + & nd & - \\
\hline Hang 2011 & + & + & + & + & - & + \\
\hline Kim 2011 & + & + & + & + & nd & - \\
\hline Komiya 2010 & + & - & + & + & - & + \\
\hline Raby 2008 & + & + & + & + & - & + \\
\hline
\end{tabular}

Table 2. Quality of studies included in this systematic review. +, good assessment; -, poor assessment; nd, not described.

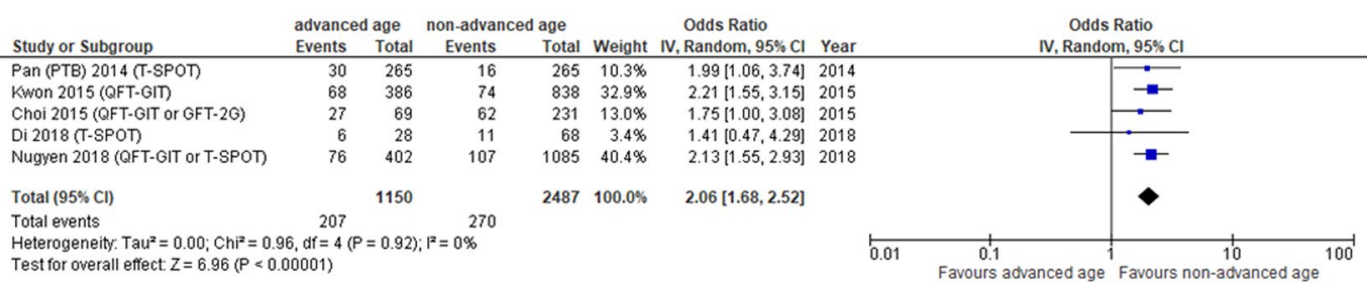

PTB; pulmonary tuberculosis

Figure 2. Forest plot for estimating the possibility of advanced age as a risk factor for false negative IGRA results.

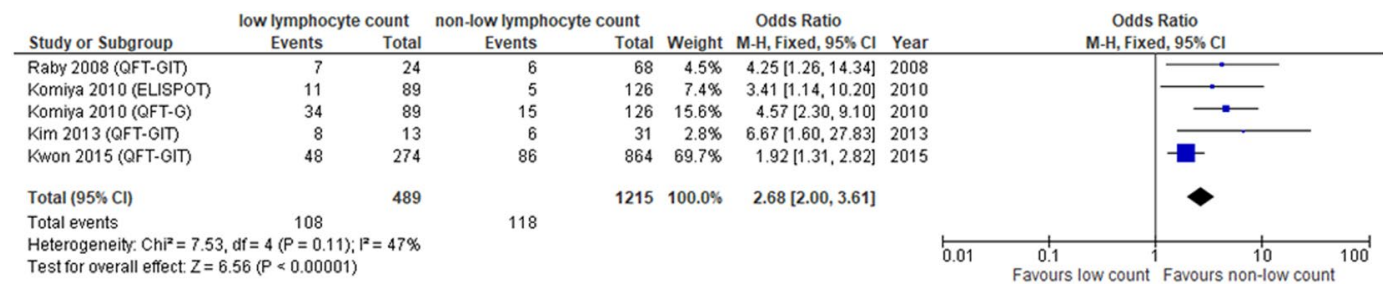

Figure 3. Forest plot for estimating the possibility of low peripheral lymphocyte counts as a risk factor for false negative IGRA results.

In the meta-analysis including raw data available in 3 of the 4 studies ${ }^{6,22,25}$, HIV infection significantly influenced false-negative results of IGRA (pooled OR 6.16, 95\% CI 1.36-27.91) with high heterogeneity (Fig. 4). Despite the pooling effect of the meta-analysis, the sample size was small in these studies, creating a large CI and thus limiting their significance. Studies from the United States ${ }^{6,22}$ and Viet $\mathrm{Nam}^{25}$ showed that HIV positivity was a significant risk factor in each multivariate analysis (see Supplementary Table S4).

Extrapulmonary TB. Five studies evaluated the influence of extrapulmonary TB on false negative results of IGRA ${ }^{15,19,21,24,26}$. QFT-GIT and T-SPOT were used for the evaluation of TB in one ${ }^{19}$ and four ${ }^{15,21,24,26}$ studies, respectively. Four ${ }^{15,21,24,26}$ studies reported that extrapulmonary TB was a significant risk factor, and all of these studies conducted assessments by T-SPOT (see Supplementary Table S5). The meta-analysis including 8 data sets from 5 studies ${ }^{15,19,21,24,26}$ in which all types of extrapulmonary TB were evaluated showed that extrapulmonary TB 


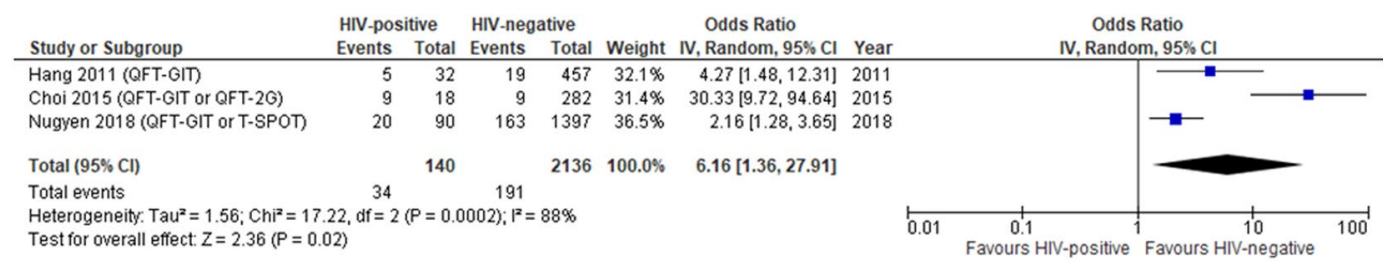

Figure 4. Forest plot for estimating the possibility of HIV positivity as a risk factor for false negative IGRA results.

(a)

\begin{tabular}{|c|c|c|c|c|c|c|c|c|c|c|}
\hline \multirow[b]{2}{*}{ Study or Subgroup } & \multicolumn{2}{|c|}{ extrapulmonary TB } & \multicolumn{2}{|c|}{ without extrapulmonary TB } & \multicolumn{3}{|c|}{ Odds Ratio } & \multirow{2}{*}{\multicolumn{2}{|c|}{$\begin{array}{c}\text { Odds Ratio } \\
\text { IV, Random, } 95 \% \mathrm{Cl}\end{array}$}} & \\
\hline & Events & Total & Events & Total & Weight & IV, Random, $95 \% \mathrm{Cl}$ & Year & & & \\
\hline Lee skeltal TB 2013 (T-SPOT) & 0 & 23 & 22 & 105 & $5.4 \%$ & $0.08[0.00,1.35]$ & 2013 & $\leftarrow$ & & \\
\hline Lee CNS TB 2013 (T-SPOT) & 10 & 36 & 12 & 92 & $13.2 \%$ & $2.56[0.99,6.62]$ & 2013 & & & \\
\hline Lian pleura TB 2017 (T-SPOT) & 23 & 251 & 54 & 305 & $15.2 \%$ & $0.47[0.28,0.79]$ & 2017 & $\rightarrow$ & & \\
\hline Lian bonerjoint TB 2017 (T-SPOT) & 10 & 22 & 67 & 534 & $13.6 \%$ & $5.81[2.42,13.97]$ & 2017 & & & \\
\hline Kim CNS TB 2018 (QFT-GIT) & 15 & 26 & 32 & 137 & $13.6 \%$ & $4.47[1.87,10.71]$ & 2018 & & & \\
\hline Kim GI TB 2018 (QFT-GIT) & 7 & 58 & 40 & 105 & $13.6 \%$ & $0.22[0.09,0.54]$ & 2018 & & & \\
\hline Di CNS TB 2018 (T-SPOT) & 6 & 8 & 11 & 90 & $9.4 \%$ & $21.55[3.86,120.34]$ & 2018 & & & \\
\hline Yang 2018 (T-SPOT) & 130 & 585 & 475 & 1840 & $16.0 \%$ & $0.82[0.66,1.02]$ & 2018 & & & \\
\hline Total $(95 \% \mathrm{Cl})$ & & 1009 & & 3208 & $100.0 \%$ & $1.44[0.64,3.25]$ & & & & \\
\hline Total events & 201 & & 713 & & & & & & & \\
\hline $\begin{array}{l}\text { Heterogeneity. } \text { Tau }^{2}=1.06 ; \mathrm{Ch}^{2}= \\
\text { Test for overall effect: } Z=0.89(P=\end{array}$ & $\begin{array}{l}19, \mathrm{df}=7(\mathrm{P} \\
37)\end{array}$ & $.00001)$ & $: 1^{2}=90 \%$ & & & & & $\begin{array}{c}0.1 \\
\text { Favours EPTB }\end{array}$ & Favours wit & $\begin{array}{l}10 \\
\text { vithout EPTB }\end{array}$ \\
\hline
\end{tabular}

(b)

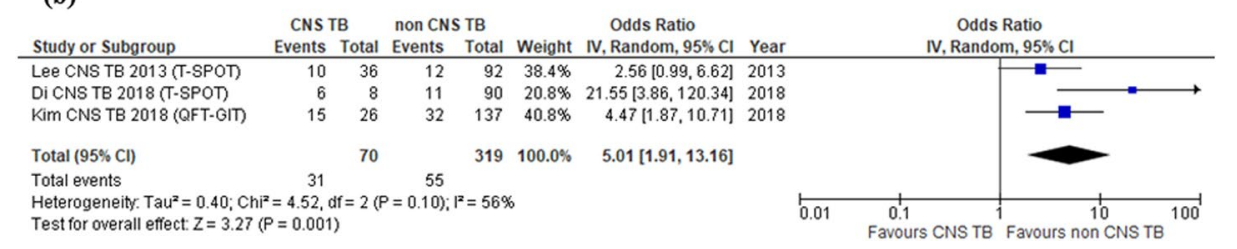

Figure 5. Forest plot for estimating the possibility of extrapulmonary TB as a whole (a) and CNS TB specifically (b) as risk factors for false negative IGRA results.

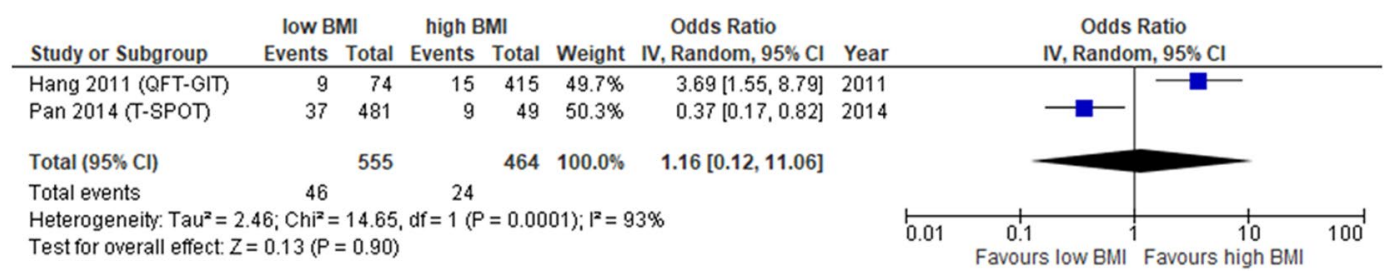

Figure 6. Forest plot for estimating the possibility of the BMI as a risk factor for false negative IGRA results.

was not a significant risk factor (pooled OR 1.44, 95\% CI 0.64-3.25) (Fig. 5a). However, when data were restricted to central nervous system (CNS) TB cases, extrapulmonary TB was indeed significantly associated with false negative results (pooled OR 5.01, 95\% CI 1.91-13.16) with moderate heterogeneity (Fig. 5b). The significance of the pooled results was limited because the studies used for this analysis included a small population. In the multivariate analyses of each study, bone or joint TB, pleural TB and CNS TB were reported to be significant risk factors even after adjusting for other variables ${ }^{15,26}$.

BMI. The BMI was studied as a risk factor for false negative results in 3 studies $^{8,23,25}$. A meta-analysis using available raw data from 2 studies ${ }^{8,25}$ showed that the BMI was not related to false negative results (pooled OR 1.16, 95\% CI 0.12-11.06) (Fig. 6). Two studies ${ }^{8,25}$ reported the significant influence of the BMI on false negative results of IGRAs in a multivariate analysis (see Supplementary Table S6). While Pan et al. found that a high BMI was significantly associated with false negative results ${ }^{8}$, Hang et al. reported the opposite finding in their multivariate analysis ${ }^{25}$.

Other factors. Smoking ${ }^{13}$, hospitalization $(>6 \text { months })^{8}$, immunosuppressive conditions ${ }^{18}$, immunosuppressive therapy ${ }^{7}$, malignancy ${ }^{20}$, high CRP ${ }^{16}$, low serum albumin $(<3.3 \mathrm{mg} / \mathrm{dl})^{18}$ and HLA type $(\mathrm{DRB} 1 * 0701 \text { alleles })^{25}$ were reported to be significant risk factors of false negative IGRA results. Although these factors were all assessed in two or more studies, statistical significance was found in only one study (see Supplementary Table S7). 
Immunosuppressive conditions as a risk factor were assessed in 11 studies 6 ,8,14,16-18,21,22,24-26. However, "immunosuppressive condition" was not clearly defined in most of the studies. Only one study ${ }^{18}$ reported an immunosuppressive condition as a significant risk factor for false negative QFT-GIT results ${ }^{18}$. Komiya et al. reported immunosuppressive therapy as a risk factor for false-negative ELISPOT results but did not describe the details of the treatment ${ }^{7}$. Kwon et al. reported malignancy as a risk factor for false negative results of QFT-GIT, but they did not describe which type of malignancy and whether or not the patients had received chemotherapy and radiotherapy ${ }^{20}$.

Two studies reported a low serum albumin level $(<3.3 \mathrm{mg} / \mathrm{dl})^{7}$ or a longer duration of illness before hospitalization due to TB (>6 months $)^{8}$ as risk factors related to false negative results. Hang et al. identified a specific HLA class II allele (DRB ${ }^{*} 0701$ allele) and reported an increased number of this allele as a risk factor for false negative results of QFT-GIT ${ }^{25}$.

\section{Discussion}

This systematic review revealed that a variety of risk factors influencing false negative results have been reported. The most commonly studied risk factor was advanced age, followed by low peripheral lymphocyte counts, and these factors were significantly associated with false negative results, regardless of TB incidence. HIV positivity and CNS TB were also likely to increase the risk of false negative results, despite being mentioned in only a limited number of studies.

Both an advanced age and low peripheral lymphocyte counts were proposed as significant risk factors for false negative results in two studies, as shown in Supplemental Tables 2 and $3^{16,20}$. However, whether or not an advanced age and low peripheral lymphocyte count are risk factors independent of each other remains unclear. Two studies using T-SPOT with optimization of the number of lymphocytes per well demonstrated that advanced age but not a low peripheral lymphocyte count was a significant factor ${ }^{8,21}$, suggesting that aging directly attenuates IFN- $\gamma$ production from a single cell as a reaction to specific TB antigens.

Low peripheral lymphocyte counts may be related to advanced age $\mathrm{e}^{27,28}$. It is reasonable that peripheral lymphocyte counts are positively correlated with the amount of IFN- $\gamma$ production in QFT but not T-SPOT, as QFT does not require the optimization of the number of lymphocytes per test. Indeed, Komiya et al. reported that the sensitivity of IGRAs depends in part on the peripheral lymphocyte count, and ELISPOT was superior to QFT for detecting TB under low-lymphocyte-count conditions ${ }^{7}$. For elderly patients with low peripheral lymphocyte counts, T-SPOT may be superior for achieving a reduced rate of false negative results. However, whether or not lymphocyte counts were a risk factor for false negative results was mainly evaluated in middle-incidence countries. Studies focusing on peripheral lymphocyte counts are needed in order to verify these results in low- or high-incidence countries as well.

$\mathrm{HIV}$ infects $\mathrm{CD}^{+}$lymphocytes and reduces the number of cells in the periphery. HIV positivity is an independent risk factor for false negative IGRA results based on total peripheral lymphocyte counts, according to the study results of Hang et al. ${ }^{25}$. They found no difference in the peripheral lymphocyte counts between the positive QFT-GIT group and the false negative group. However, there is no evidence concerning whether or not HIV positivity is independent of the $\mathrm{CD} 4^{+}$lymphocyte counts.

Some studies have suggested that the site of TB infection may significantly affect the T-SPOT sensitivity ${ }^{14,29,30}$. The infection site may therefore also be associated with false negative results of IGRA. While the exact reason why the T-cell response of IGRA differs among organs is unclear, CNS TB was found to be a significant risk factor for false negative IGRA results in the pooled analysis shown in Fig. 5b. The sensitivity of culture and polymerase chain reaction (PCR) of $M$. tuberculosis from cerebral spinal fluid is very low in patients with TB meningitis ${ }^{31-33}$. Given that the blood brain barrier does not allow the components of bacilli to penetrate to the CNS ${ }^{33}$, the frequency of lymphocytes contacting specific antigens in the CNS may be lower than that in the lung, which may explain the increased rate of false negative IGRA results.

The BMI and serum albumin level are usually considered to reflect the nutritional status. For example, a low BMI may indicate malnutrition or severe wasting disease. These conditions may suppress the systemic immune response $\mathrm{e}^{34}$ and reduce lymphocyte reactions to TB specific antigens on QFT-GIT. Pan et al. reported that a high BMI was a significant risk factor for false negative T-SPOT test ${ }^{8}$, although the reasons for this high false negative rate are not fully discussed in the article. Diabetes mellitus (DM) is often concomitant with a high BMI. Faurholt-Jepsen et al. reported that the IFN- $\gamma$ level of QFT was reduced in DM patients with and without $\mathrm{TB}^{35}$. This may explain the relationship between a high BMI and the increased risk of false negative results. However, Pan et al. also showed that DM was not a significant risk factor in the same population. Another study found that the sensitivity of both T-SPOT and QFT was not affected by $\mathrm{DM}^{36}$. The influence of both high BMI and DM on the sensitivity therefore remains controversial.

Immunosuppressive conditions were assessed in 11 studies, and only 1 found significance in a multivariate analysis. Immunosuppressive conditions may be characterized by immunosuppressive therapy, malignancy and likely DM, but no clear definition exists. Therefore, this vague categorization should not be applied for studies assessing the sensitivity of immunological tests; instead, each factor needs to be evaluated separately.

The strength of our study was that we systematically reviewed for the first time the risk factors for false negative results of IGRA with a meta-analysis. However, this systematic review also has several limitations. First, the definitions and cut-off values for each factor varied among the included studies. Common definitions are needed in order to evaluate the impact of each factor more objectively. Second, small-scale studies were included in this systematic review in order to collect as much data as possible. This might generate low heterogeneity, so conducting a large-scale study is encouraged. Finally, whether or not the IGRA was conducted in in-house or commercially, which can cause measurement bias, was unclear in most studies. The IGRA results may be influenced by the handling of samples or the procedures of IGRA testing, in addition to host factors. 
This systematic review suggested that advanced age and low peripheral lymphocyte counts were most likely to be associated with false negative IGRA results. Assessments of risk factors for false negative IGRA results with common definitions and the same cut-off values (e.g. advanced age) are needed. Once more reliable evidence is obtained in future studies, the next step will be to create a strategy for reducing false negative results. Substantial variability with repeated IGRA testing was reported, even under identical conditions. This suggests that reversions and conversions around the existing cut-off point should be interpreted with caution ${ }^{37,38}$. Standardization of the assay procedure, including the interval time from blood sampling to the analysis, as well as reconsideration of the best cut-off value by groups with individual risks of false negative IGRA results while considering the reproducibility of the results is required.

Received: 11 September 2019; Accepted: 9 January 2020;

Published online: 31 January 2020

\section{References}

1. World Health Organization. Grobal tuberculosis report 2018. Geneva, World Health Organization, 2018 (2018).

2. Diel, R., Loddenkemper, R. \& Nienhaus, A. Predictive value of interferon-gamma release assays and tuberculin skin testing for progression from latent TB infection to disease state: a meta-analysis. Chest 142, 63-75, https://doi.org/10.1378/chest.11-3157 (2012).

3. Rangaka, M. X. et al. Predictive value of interferon-gamma release assays for incident active tuberculosis: a systematic review and meta-analysis. Lancet. Infect. Dis. 12, 45-55, https://doi.org/10.1016/s1473-3099(11)70210-9 (2012).

4. Sester, M. et al. Interferon-gamma release assays for the diagnosis of active tuberculosis: a systematic review and meta-analysis. Eur. respiratory J. 37, 100-111, https://doi.org/10.1183/09031936.00114810 (2011).

5. World Health Organization. Latent tuberculosis infection: updated and consolidated guidelines for programmatic management. Geneva, World Health Organization, 2018 (2018).

6. Choi, J. C. et al. Reduced sensitivity of the QuantiFERON $((\mathrm{R}))$ test in diabetic patients with smear-negative tuberculosis. Int. J. Tuberc. Lung Dis. 19, 582-588, https://doi.org/10.5588/ijtld.14.0553 (2015).

7. Komiya, K. et al. Impact of peripheral lymphocyte count on the sensitivity of 2 IFN-gamma release assays, QFT-G and ELISPOT, in patients with pulmonary tuberculosis. Intern. Med. 49, 1849-1855 (2010).

8. Pan, L. et al. Risk factors for false-negative T-SPOT.TB assay results in patients with pulmonary and extra-pulmonary TB. J. Infect. 70, 367-380, https://doi.org/10.1016/j.jinf.2014.12.018 (2015).

9. Moher, D., Liberati, A., Tetzlaff, J. \& Altman, D. G. Preferred reporting items for systematic reviews and meta-analyses: the PRISMA statement. PLoS Med. 6, e1000097, https://doi.org/10.1371/journal.pmed.1000097 (2009).

10. Stroup, D. F. et al. Meta-analysis of observational studies in epidemiology: a proposal for reporting. Meta-analysis Of Observational Studies in Epidemiology (MOOSE) group. Jama 283, 2008-2012 (2000).

11. Hayden, J. A., Cote, P. \& Bombardier, C. Evaluation of the quality of prognosis studies in systematic reviews. Ann. Intern. Med. 144, 427-437 (2006).

12. Sterne, J. A., Egger, M. \& Smith, G. D. Systematic reviews in health care: Investigating and dealing with publication and other biases in meta-analysis. BMJ 323, 101-105 (2001).

13. Aabye, M. G. et al. Negative effect of smoking on the performance of the QuantiFERON TB gold in tube test. BMC Infect. Dis. 12, 379, https://doi.org/10.1186/1471-2334-12-379 (2012).

14. de Visser, V. et al. False-negative interferon-gamma release assay results in active tuberculosis: a TBNET study. Eur. respiratory J. 45, 279-283, https://doi.org/10.1183/09031936.00120214 (2015).

15. Di, L. \& Li, Y. The risk factor of false-negative and false-positive for T-SPOT.TB in active tuberculosis. J Clin Lab Anal 32, https://doi. org/10.1002/jcla.22273 (2018).

16. Jeon, Y. L. et al. Factors influencing discordant results of the QuantiFERON-TB Gold In-tube test in patients with active TB. J. Infect. 67, 288-293, https://doi.org/10.1016/j.jinf.2013.06.005 (2013).

17. Kim, C. H. et al. Diagnostic performance of the QuantiFERON-TB Gold In-Tube assay and factors associated with nonpositive results in patients with miliary tuberculosis. Clin. Infect. diseases: an. Off. Publ. Infect. Dis. Soc. Am. 58, 986-989, https://doi. org/10.1093/cid/ciu045 (2014).

18. Kim, E. Y. et al. Risk factors for false-negative results of QuantiFERON-TB Gold In-Tube assay in non-HIV-infected patients with culture-confirmed tuberculosis. Diagn. Microbiol. Infect. Dis. 70, 324-329, https://doi.org/10.1016/j.diagmicrobio.2011.02.011 (2011).

19. Kim, Y. J. et al. Predictors for false-negative QuantiFERON-TB Gold assay results in patients with extrapulmonary tuberculosis. BMC Infect. Dis. 18, 457, https://doi.org/10.1186/s12879-018-3344-x (2018).

20. Kwon, Y. S. et al. Factors that Predict Negative Results of QuantiFERON-TB Gold In-Tube Test in Patients with Culture-Confirmed Tuberculosis: A Multicenter Retrospective Cohort Study. PLoS One 10, e0129792, https://doi.org/10.1371/journal.pone.0129792 (2015).

21. Lian, G., Du, F., Wu, H., He, M. \& Liu, Z. Factors Contributing to False-Negative Enzyme-Linked Immunospot Assay for InterferonGamma Results in Active Tuberculosis. Clin. Lab. 63, 773-779, https://doi.org/10.7754/Clin.Lab.2016.161007 (2017).

22. Nguyen, D. T., Teeter, L. D., Graves, J. \& Graviss, E. A. Characteristics Associated with Negative Interferon-gamma Release Assay Results in Culture-Confirmed Tuberculosis Patients, Texas, USA, 2013-2015. Emerg. Infect. Dis. 24, 534-540, https://doi. org/10.3201/eid2403.171633 (2018).

23. Raby, E. et al. The effects of HIV on the sensitivity of a whole blood IFN-gamma release assay in Zambian adults with active tuberculosis. PLoS One 3, e2489, https://doi.org/10.1371/journal.pone.0002489 (2008).

24. Yang, C., Zhang, S., Yao, L. \& Fan, L. Evaluation of risk factors for false-negative results with an antigen-specific peripheral bloodbased quantitative T cell assay (T-SPOT((R)). TB) in the diagnosis of active tuberculosis: A large-scale retrospective study in China. J. Int. Med. Res. 46, 1815-1825, https://doi.org/10.1177/0300060518757381 (2018).

25. Hang, N. T. et al. Analysis of factors lowering sensitivity of interferon-gamma release assay for tuberculosis. PLoS One 6, e23806, https://doi.org/10.1371/journal.pone.0023806 (2011).

26. Lee, Y. M. et al. Risk factors for false-negative results of T-SPOT.TB and tuberculin skin test in extrapulmonary tuberculosis. Infect. 41, 1089-1095, https://doi.org/10.1007/s15010-013-0478-z (2013).

27. Pinti, M. et al. Aging of the immune system: Focus on inflammation and vaccination. Eur. J. immunology 46, 2286-2301, https://doi. org/10.1002/eji.201546178 (2016).

28. Franceschi, C., Monti, D., Sansoni, P. \& Cossarizza, A. The immunology of exceptional individuals: the lesson of centenarians. Immunology today 16, 12-16 (1995).

29. Cho, O. H. et al. Diagnostic performance of T-SPOT.TB for extrapulmonary tuberculosis according to the site of infection. J. Infect. 63, 362-369, https://doi.org/10.1016/j.jinf.2011.06.010 (2011). 
30. Liao, C. H. et al. False-negative results by enzyme-linked immunospot assay for interferon-gamma among patients with cultureconfirmed tuberculosis. J. Infect. 59, 421-423, https://doi.org/10.1016/j.jinf.2009.09.012 (2009).

31. Erdem, H. et al. The microbiological diagnosis of tuberculous meningitis: results of Haydarpasa-1 study. Clin. microbiology infection: Off. Publ. Eur. Soc. Clin. Microbiology Infect. Dis. 20, O600-608, https://doi.org/10.1111/1469-0691.12478 (2014).

32. Pai, M. et al. Diagnostic accuracy of nucleic acid amplification tests for tuberculous meningitis: a systematic review and metaanalysis. Lancet. Infect. Dis. 3, 633-643 (2003).

33. van Leeuwen, L. M. et al. Mycobacteria employ two different mechanisms to cross the blood-brain barrier. Cell. microbiology 20 , e12858, https://doi.org/10.1111/cmi.12858 (2018).

34. Schluger, N. W. \& Rom, W. N. The host immune response to tuberculosis. Am. J. respiratory Crit. care Med. 157, 679-691, https:// doi.org/10.1164/ajrccm.157.3.9708002 (1998).

35. Faurholt-Jepsen, D. et al. Diabetes is associated with lower tuberculosis antigen-specific interferon gamma release in Tanzanian tuberculosis patients and non-tuberculosis controls. Scand. J. Infect. Dis. 46, 384-391, https://doi.org/10.3109/00365548.2014.8856 57 (2014).

36. Gan, S. H. et al. Interferon-gamma responses to Mycobacterium tuberculosis-specific antigens in diabetes mellitus. Eur. respiratory J. 44, 805-808, https://doi.org/10.1183/09031936.00226613 (2014).

37. Banaei, N., Gaur, R. L. \& Pai, M. Interferon Gamma Release Assays for Latent Tuberculosis: What Are the Sources of Variability? J. Clin. microbiology 54, 845-850, https://doi.org/10.1128/jcm.02803-15 (2016).

38. Tagmouti, S. et al. Reproducibility of interferon gamma (IFN-gamma) release Assays. A systematic review. Ann. Am. Thorac. Soc. 11, 1267-1276, https://doi.org/10.1513/AnnalsATS.201405-188OC (2014).

\section{Acknowledgements}

This research did not receive any specific grant from funding agencies in the public, commercial, or not-for-profit sectors.

\section{Author contributions}

M.Y., K.K. Y.U., K.U., S.N., M.A., K.H. H.N. and J.K. reviewed and prepared the manuscript. M.Y., K.K., H.N. and J.K. contributed to the protocol design. M.Y. and K.K. performed manuscript screening, data extraction and analyses.

\section{Competing interests}

The authors declare no competing interests.

\section{Additional information}

Supplementary information is available for this paper at https://doi.org/10.1038/s41598-020-58459-9.

Correspondence and requests for materials should be addressed to K.K.

Reprints and permissions information is available at www.nature.com/reprints.

Publisher's note Springer Nature remains neutral with regard to jurisdictional claims in published maps and institutional affiliations.

(c) (i) Open Access This article is licensed under a Creative Commons Attribution 4.0 International License, which permits use, sharing, adaptation, distribution and reproduction in any medium or format, as long as you give appropriate credit to the original author(s) and the source, provide a link to the Creative Commons license, and indicate if changes were made. The images or other third party material in this article are included in the article's Creative Commons license, unless indicated otherwise in a credit line to the material. If material is not included in the article's Creative Commons license and your intended use is not permitted by statutory regulation or exceeds the permitted use, you will need to obtain permission directly from the copyright holder. To view a copy of this license, visit http://creativecommons.org/licenses/by/4.0/.

(C) The Author(s) 2020 